Spin Physics (SPIN2014)

International Journal of Modern Physics: Conference Series

Vol. 40 (2016) 1660055 (6 pages)

(C) The Author(s)

DOI: 10.1142/S2010194516600557

\title{
Wigner Distributions of Quark
}

\author{
Asmita Mukherjee*, Sreeraj Nair ${ }^{\dagger}$ and Vikash Kumar Ojha ${ }^{\ddagger}$ \\ Department of Physics, Indian Institute of Technology Bombay, \\ Powai, Mumbai 400076, India \\ *asmita@phy.iitb.ac.in

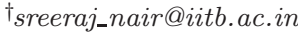 \\ ${ }_{\ddagger}^{\ddagger}$ vikashojha@iitb.ac.in
}

Published 29 February 2016

\begin{abstract}
Wigner distribution functions are the quantum analogue of the classical phase space distribution and being quantum implies that they are not genuine phase space distribution and thus lack any probabilistic interpretation. Nevertheless, Wigner distributions are still interesting since they can be related to both generalized parton distributions (GPDs) and transverse momentum dependent parton distributions (TMDs) under some limit. We study the Wigner distribution of quarks and also the orbital angular momentum (OAM) of quarks in the dressed quark model.
\end{abstract}

Keywords: Wigner; quark; orbital angular momentum.

\section{Introduction}

Wigner functions were first introduced by E.P. Wigner ${ }^{1}$ in an attempt to provide quantum corrections to classical statistical mechanics thereby providing a probability distribution for a quantum state. Wigner functions are not genuine phase-space distribution function because of the uncertainty principle and the fact that they are not positive definite. Wigner distribution for the quarks and gluons in the rest frame of the nucleon were introduced in Refs. 2 and 3. Wigner distributions are related to the generalized transverse momentum dependent parton distributions (GTMDs) which are off-diagonal quark-quark correlators obtained by integrating out the lightcone energy $\left(k^{-}\right)$from the fully unintegrated correlators. Wigner distributions in infinite momentum frame obtained by integrating the GTMDs over the momentum transfer in the transverse direction $\Delta_{\perp}$ were introduced in Ref. 4 . These Wigner distributions are functions of the two position and three momentum variables. In

This is an Open Access article published by World Scientific Publishing Company. It is distributed under the terms of the Creative Commons Attribution 3.0 (CC-BY) License. Further distribution of this work is permitted, provided the original work is properly cited. 
Ref. 4 the Wigner distributions for quarks and gluons have been studied in models devoid of gluonic degrees of freedom namely the light cone constituent quark model and the light-cone chiral quark soliton model.

After the EMC experiments ${ }^{5}$ it was clear that we need to have a better understanding of the orbital angular momentum (OAM) of partons inside the nucleon because the experiment showed that the contribution of the quark intrinsic spin to the total spin half of the nucleon was substantially less than what was expected. So as far as we know the only remaining candidate which could account for the missing spin were the OAM of quarks and the total spin angular momentum of the gluons. The Wigner distributions are related to the OAM carried by the quarks in the nucleon.

Here we present a calculation of the quark Wigner distributions in light-front Hamiltonian formulation using overlaps of light-front wave functions (LFWFs) which is based on the approach shown in Ref. 6. We calculate the Wigner distributions and also the OAM for a quark dressed with a gluon in light-front Hamiltonian approach.

\section{Wigner Distributions}

Starting from the GTMDs, ${ }^{4,7}$ we can define the quark Wigner distributions as a two-dimensional Fourier transform with respect to the transverse momentum transfer $\Delta_{\perp}$.

$$
\rho\left(\vec{b}_{\perp}, \vec{k}_{\perp}, x, \sigma\right)=\int \frac{d^{2} \Delta_{\perp}}{(2 \pi)^{2}} e^{-i \Delta_{\perp} . b_{\perp}} W\left(\Delta_{\perp}, \vec{k}_{\perp}, x, \sigma\right) ;
$$

where $\Delta_{\perp}$ is the momentum transfer of the dressed quark in the transverse direction and $\vec{b}_{\perp}$ is the 2 dimensional vector in the impact parameter space conjugate to $\Delta_{\perp}$. $W$ is the quark-quark correlator given by

$$
\begin{aligned}
W\left(\vec{\Delta}_{\perp}, \vec{k}_{\perp}, x, \sigma\right)= & \left\langle p^{+}, \frac{\Delta_{\perp}}{2}, \sigma\left|W\left(0_{\perp}, k_{\perp}, x\right)\right| p^{+},-\frac{\Delta_{\perp}}{2}, \sigma\right\rangle \\
= & \frac{1}{2} \int \frac{d z^{-} d^{2} z \perp}{(2 \pi)^{3}} e^{i\left(x p^{+} z^{-} / 2-k_{\perp}, z_{\perp}\right)} \\
& \times\left.\left\langle p^{+}, \frac{\Delta_{\perp}}{2}, \sigma\left|\bar{\psi}\left(-\frac{z}{2}\right) \gamma^{+} \psi\left(\frac{z}{2}\right)\right| p^{+},-\frac{\Delta_{\perp}}{2}, \sigma\right\rangle\right|_{z^{+}=0} .
\end{aligned}
$$

We use the symmetric frame as shown in Ref. 8 for defining the initial and final dressed quark state. In the symmetric frame the transverse momentum transfer $\left(\Delta_{\perp}\right)$ has the $\Delta_{\perp} \rightarrow-\Delta_{\perp}$ symmetry. $p^{+}$and $\sigma$ define the longitudinal momentum of the target state and its helicity respectively. $x=k^{+} / p^{+}$is the fraction of longitudinal momentum of the dressed quark carried by the quark. We consider the Dirac operator to be $\gamma^{+}$and work in the light-front gauge thereby setting the gauge link to unity.

We expand the states in Fock space in terms of multi-parton light-front wave functions $(\mathrm{LFWFs})^{9}$ and calculate the contribution coming from the two particle 
sector in the Fock space expansion. The final expression for the Wigner distribution in terms of the two particle LFWF is

$$
W\left(\Delta_{\perp}, k_{\perp}, x, \sigma\right)=\frac{1}{(2 \pi)^{3}} \sum_{\sigma_{1}, \sigma_{2}} \Psi_{\sigma_{1} \sigma_{2}}^{* \sigma a}\left(x, q_{\perp}^{\prime}\right) \Psi_{\sigma_{1} \sigma_{2}}^{\sigma a}\left(x, q_{\perp}\right) .
$$

With $\gamma^{+}$as the Dirac operator sandwiched between the quark fields we can define two Wigner distributions ${ }^{4}$

$$
\rho_{U U}\left(\vec{b}_{\perp}, \vec{k}_{\perp}, x\right)=\frac{1}{2}\left[\rho\left(\vec{b}_{\perp}, \vec{k}_{\perp}, x,+\vec{e}_{z}\right)+\rho\left(\vec{b}_{\perp}, \vec{k}_{\perp}, x,-\vec{e}_{z}\right)\right]
$$

is the Wigner distribution of unpolarized quarks in unpolarized target state.

$$
\rho_{L U}\left(\vec{b}_{\perp}, \vec{k}_{\perp}, x\right)=\frac{1}{2}\left[\rho\left(\vec{b}_{\perp}, \vec{k}_{\perp}, x,+\vec{e}_{z}\right)-\rho\left(\vec{b}_{\perp}, \vec{k}_{\perp}, x,-\vec{e}_{z}\right)\right]
$$

is the distortion due to longitudinal polarization of the target state. $+\overrightarrow{e_{z}}$ and $-\overrightarrow{e_{z}}$ correspond to helicity up and down of the target state and the final expressions for the above two Wigner distributions are

$$
\begin{gathered}
\rho_{U U}\left(b_{\perp}, k_{\perp}, x\right)=\int_{-a_{x}}^{a_{x}} d \Delta_{x} \int_{-a_{y}}^{a_{y}} d \Delta_{y} \frac{\cos \left(\Delta_{\perp} \cdot b_{\perp}\right)}{D\left(q_{\perp}\right) D\left(q_{\perp}^{\prime}\right)}\left[I_{1}+\frac{4 m^{2}(1-x)}{x^{2}}\right] ; \\
\rho_{L U}\left(b_{\perp}, k_{\perp}, x\right)=\int_{-a_{x}}^{a_{x}} d \Delta_{x} \int_{-a_{y}}^{a_{y}} d \Delta_{y} \frac{\sin \left(\Delta_{\perp} \cdot b_{\perp}\right)}{D\left(q_{\perp}\right) D\left(q_{\perp}^{\prime}\right)}\left[4\left(k_{x} \Delta_{y}-k_{y} \Delta_{x}\right) \frac{(1+x)}{x^{2}(1-x)}\right] .
\end{gathered}
$$

where $A_{x}, A_{y}$ are $x, y$ component of $A_{\perp}$ and

$$
\begin{gathered}
D\left(k_{\perp}\right)=\left(m^{2}-\frac{m^{2}+\left(k_{\perp}\right)^{2}}{x}-\frac{\left(k_{\perp}\right)^{2}}{1-x}\right) \quad I_{1}=4\left(\left(k_{\perp}\right)^{2}-\frac{\Delta_{\perp}^{2}(1-x)^{2}}{4}\right) \frac{\left(1+x^{2}\right)}{x^{2}(1-x)^{3}} . \\
a_{x}=\Delta_{x}^{\max }=\Delta_{\max } \sin \theta \quad a_{y}=\Delta_{y}^{\max }=\Delta_{\max } \cos \theta
\end{gathered}
$$

For all the plots we have set $\theta=45^{\circ}$ in the integration limits. In Fig. 1 we show the contour plots for the Wigner distribution $\rho_{U U}$ and $\rho_{L U}$ in $b$ space and $k$ space respectively. Since we are integrating from $-a_{i}$ to $a_{i}$ where $i=x, y$ in Eq. (6) we observe an asymmetry associated with the orbital angular momentum for $\rho_{U U}$ similar to that observed in Ref. 4. This asymmetry favors the $b \perp k$ direction in comparison to the $b \| k$ direction. $\rho_{U U}$ has a peak centered at $b_{x}=b_{y}=0$ decreasing in the outer regions of the $b$ space. In Fig. $1 b$ the contour plot represents the distortion of the Wigner distribution of unpolarized quarks due to the longitudinal polarization of the dressed quark. Like in Ref. 4 we observe a dipole structure for $\rho_{L U}$ in $k$ space.

In Fig. 2 (a) and (b) we show the plots in the mixed space. Wigner distributions do not have probability interpretation due to uncertainty principle in quantum mechanics but in the distributions $\rho_{U U}\left(k_{y}, b_{x}\right)$ and $\rho_{L U}\left(k_{y}, b_{x}\right)$ we have integrated out the $k_{x}$ and $b_{y}$ dependence giving us the probability densities correlating $k_{y}$ and $b_{x}$, this correlation is not restricted by uncertainty principle. 

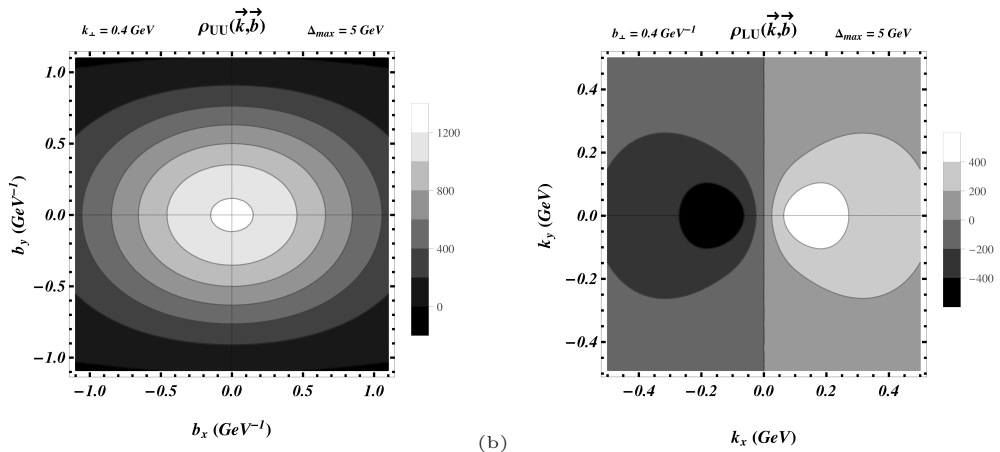

Fig. 1. Contour plots of the Wigner distributions where plot (a) is for $\rho_{U U}$ in $b$ space with $k_{\perp}=0.4 \mathrm{GeV}$ and (b) is for $\rho_{L U}$ in $k$ space with $b_{\perp}=0.4 \mathrm{GeV}^{-1}$. For both the plots we kept $\Delta_{\max }=5.0 \mathrm{GeV}, m=0.33 \mathrm{GeV}$ and integrated out the $x$ variable and we took $\overrightarrow{k_{\perp}}=k \hat{j}$ and $\overrightarrow{b_{\perp}}=b \hat{j}$.
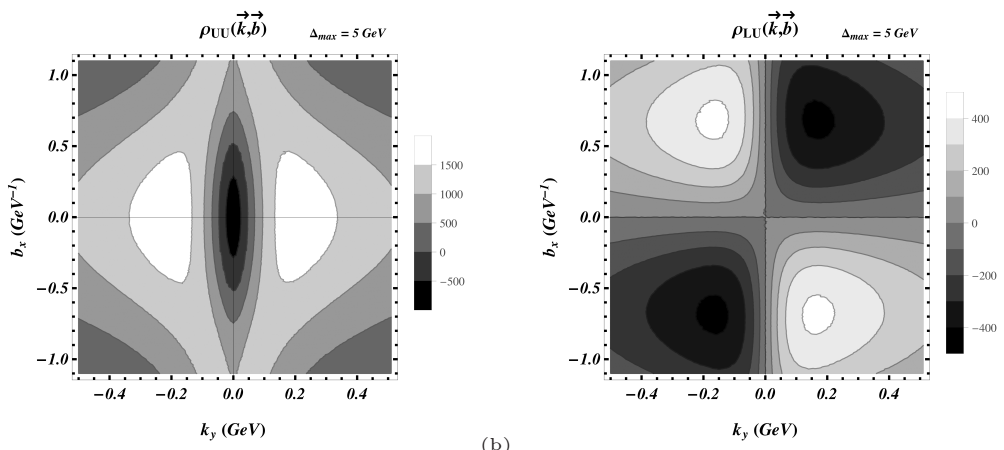

(a)

Fig. 2. Contour plots of the Wigner distributions in the mixed space where $k_{x}$ and $b_{y}$ are integrated. Plot (a) is for $\rho_{U U}$ and (b) is for $\rho_{L U}$. For both the plots we kept $\Delta_{\max }=5.0 \mathrm{GeV}$, $m=0.33 \mathrm{GeV}$ and integrated out the $x$ variable.

In Fig. 2 (a) we observe a minima at $b_{x}=0$ and $k_{y}=0$ unlike that in Ref. 4. In fact the minima is observed for all $b_{x}$ values for $k_{y}=0$. The plots show that the probability of finding a quark with fixed $k_{y}$ and $b_{x}$ first increases away from $k_{y}=0$ and then decreases. In Fig. 2 (b) we observe the quadrupole structure in the mixed space like in Ref. 4.

\section{Orbital Angular Momentum of Quarks}

The kinetic quark orbital angular momentum ${ }^{10}(\mathrm{OAM})$ is given in terms of the GPDs as:

$$
L_{z}^{q}=\frac{1}{2} \int d x\left\{x\left[H^{q}(x, 0,0)+E^{q}(x, 0,0)\right]-\tilde{H}^{q}(x, 0,0)\right\} .
$$


The kinetic OAM is related to the $\mathrm{GTMDs}^{7}$ and the quark-quark correlator in Eq.(2) defining the Wigner distributions can be parametrized in terms of generalized transverse momentum dependent parton distributions $\left(\right.$ GTMDs) ${ }^{7}$ and for the twist two case we have four GTMDs $\left(F_{1, i}\right)$ corresponding to the Dirac operator $\gamma^{+}$and four more for $\gamma^{+} \gamma_{5}\left(G_{1, i}\right)$. We can get an expression for the OAM by calculating these 8 GTMDs in the dressed quark model.

So the final expression for the kinetic orbital angular momentum of quarks in the dressed quark model is:

$$
L_{z}^{q}=\frac{N}{2} \int d x\left\{-f(x) I_{1}+4 m^{2}(1-x)^{2} I_{2}\right\}
$$

where,

$$
\begin{aligned}
I_{1} & =\int \frac{d^{2} k_{\perp}}{m^{2}(1-x)^{2}+\left(k_{\perp}\right)^{2}}=\pi \log \left[\frac{Q^{2}+m^{2}(1-x)^{2}}{\mu^{2}+m^{2}(1-x)^{2}}\right] ; \\
I_{2} & =\int \frac{d^{2} k_{\perp}}{\left(m^{2}(1-x)^{2}+\left(k_{\perp}\right)^{2}\right)^{2}}=\frac{\pi}{\left(m^{2}(1-x)^{2}\right)} ; \\
f(x) & =2\left(1+x^{2}\right) .
\end{aligned}
$$

Here $Q$ and $\mu$ are the upper and lower limits of the $k_{\perp}$ integration respectively.

$F_{14}$ is related to the canonical OAM as shown in Refs. 4, 11 and 12

$$
l_{z}^{q}=-\int d x d^{2} k_{\perp} \frac{k_{\perp}^{2}}{m^{2}} F_{14} .
$$

The final expression for the canonical quark OAM in the dressed quark model is:

$$
l_{z}^{q}=-2 N \int d x\left(1-x^{2}\right)\left[I_{1}-m^{2}(x-1)^{2} I_{2}\right]
$$

\section{Conclusion}

We calculated the Wigner distributions using the overlap representation in terms of the LFWFs for a quark state dressed with a gluon which is a simple composite spin-1/2 system having a gluonic degree of freedom. We showed a comparative study of our results with an earlier calculation of Wigner distributions in light cone constituent quark model and light-cone chiral quark soliton model. We also calculated the kinetic quark OAM using the GPD sum rule and the canonical OAM. Because of the presence of the gluonic degree of freedom in our model, the two OAM differed in magnitude.

\section{Acknowledgments}

This work is supported by the DST project SR/S2/HEP-029/2010, Govt. of India. SN would like to thank C.Lorce for helpful discussion and also the organizers of Spin2014, October 20-24, 2014, Beijing, China for the invitation. 


\section{References}

1. E.P. Wigner, Phys. Rev. 40, 749 (1932).

2. X. Ji, Phys. Rev. Lett. 91, 062001 (2003).

3. A. Belitsky, X. Ji and F. Yuan, Phys. Rev. D 69, 074014 (2004).

4. C. Lorce and B.Pasquini, Phys. Rev. D84, 014015 (2011).

5. J. Ashman et al., Nucl. Phys. B328, 1 (1989).

6. W-M. Zhang and Harindranath, Phys. Rev. D48, 4881 (1993).

7. S. Meissner, A. Metz and M. Schlegel, JHEP. 08, 056 (2009); S.Meissner, A.Metz, M. Schlegel and K. Goeke, JHEP. 08, 038 (2008) .

8. S. J. Brodsky, M. Diehl and D. S. Hwang, Nucl. Phys. B596, 99, (2001).

9. A. Harindranath and R. Kundu, Phys. Rev. D59, 116013 (1999).

10. X. Ji, Phys. Rev. Lett. 78, 610 (1997).

11. Y. Hatta, Phys. Lett. B 708, 186 (2012).

12. C. Lorce, B. Pasquini, X. Xiong and F. Yuan, Phys. Rev. D 85, 114006 (2012). 